\title{
Biocompatibilidad y toxicidad de nanopartículas de dióxido de titanio en la cavidad oral: Revisión sistemática.
}

\author{
Liliana Argueta-Figueroa ${ }^{1}$, Nayely Torres-Góme: ${ }^{2}$, Rogelio J. Scougall-Vilchis ${ }^{3}$ \\ y René García-Contreras ${ }^{4}$ \\ ${ }^{1}$ Cátedra CONACyT, Facultad de Odontología, Universidad Autónoma Benito Juárez \\ de Oaxaca, Oaxaca, México. \\ ${ }^{2}$ Centro Conjunto de Investigación en Química Sustentable (CCIQS), UEAMex-UNAM. \\ Km 14.5, Carr. Toluca-Atlacomulco, Toluca, Estado de México, México. \\ ${ }^{3}$ Centro de Investigación y Estudios Avanzados en Odontología (CIEAO) "Dr. Keisaburo \\ Miyata", Universidad Autónoma del Estado de México (UAEMex), Toluca, México. \\ ${ }^{4}$ Laboratorio de Investigación Interdisciplinaria (LII), Área de Nanoestructuras \\ y Biomateriales, Escuela Nacional de Estudios Superiores Unidad León; Universidad \\ Nacional Autónoma de México; León, Guanajuato, México.
}

Palabras clave: aplicaciones biomédicas; citotoxicidad; viabilidad celular; prostaǵlandina $\mathrm{E}_{2}$; estrés oxidativo.

Resumen. El presente artículo tiene como propósito analizar el impacto biológico y la toxicidad de las nanopartículas (NPs) de dióxido de titanio $\left(\mathrm{TiO}_{2}\right)$ en la producción de especies reactivas de oxígeno así como inducción de estrés oxidativo, a través de una revisión sistemática de la bibliografía publicada hasta el momento sobre la biocompatibilidad de las NPs en contacto con células orales. Los datos disponibles sobre las NPs de $\mathrm{TiO}_{2}$ fueron recopilados de la base de datos electrónica PubMed, ScienceDirect y otras fuentes, de acuerdo con recomendaciones PRISMA para revisiones sistemáticas. En el análisis cualitativo de la información publicada sobre la citotoxicidad en células orales, se ha observado un ligero aumento en el número de células metabólicamente activas al estar en contacto con las NPs de $\mathrm{TiO}_{2}$; sin embargo a ciertas dosis la viabilidad celular decrece de manera importante. Uno de los efectos negativos de las $\mathrm{NPs}_{\mathrm{de}} \mathrm{TiO}_{2}$ es la inducción de producción de prostaglandina $\mathrm{E}_{2}$ en un estado proinflamatorio previo, lo que potenciaría el efecto inflamatorio ya existente. Por tal motivo, la aplicación biológica de estas NPs debe ser sujeta a estudios in vivo y metabolómicos, ya que es posible que los resultados de los experimentos in vitro no puedan ser extrapolados a un sistema in vivo debido a la tendencia a agregarse en el medio de cultivo empleado para dichas pruebas. Por lo tanto, aunque puede clasificarse como un material con citotoxidad ligera o incluso biocompatible, no puede ser considerado complemente inocuo.

Autor de correspondencia: René García-Contreras. Laboratorio de Investigación Interdisciplinaria (LII), Área de Nanoestructuras y Biomateriales, Escuela Nacional de Estudios Superiores Unidad León; Universidad Nacional Autónoma de México; León, Guanajuato, México. e-mail: dentist.garcia@gmail.com 


\title{
Biocompatibility and nanotoxicology of titanium dioxide in the oral cavity: Systematic review.
}

\author{
Invest Clin 2018; 59 (4): 352 - 368
}

Key words: biomedical applications; cytotoxicity; cell viability; prostaǵlandin $\mathrm{E}_{2}$; oxidative stress.

\begin{abstract}
The aim of the present review article was to analyze the biological impact and toxicology of titanium dioxide nanoparticles $\left(\mathrm{TiO}_{2} \mathrm{NPs}\right)$ to determine their cytotoxicity, reactive oxygen species production as well as induction of oxidative stress through a systematic review of the literature published so far about the biocompatibility of $\mathrm{TiO}_{2} \mathrm{NPs}$ in contact with oral cells. Available data on nanoparticles (NPs) were collected from the PubMed and Science Direct electronic databases, and other sources according to PRISMA recommendations for systematic reviews. In the qualitative analysis of published data on cytotoxicity in oral cells, a slight increase in the number of metabolically active cells has been observed when the $\mathrm{TiO}_{2}$ NPs are in contact with oral cells; however, at certain doses cellular viability decreases significantly. One of the negative effects of NPs is the induction of prostaglandin $\mathrm{E}_{2}$ production in a previous pro-inflammatory state, which could increase the existing inflammatory effect. Therefore, the biological application of these nanoparticles must be performed both in vivo and metabolomic studies, since it is possible that the results of the in vitro experiments cannot be extrapolated to an in vivo system, since it been observed that the particles tend to aggregated among them, in the culture medium, used for such as tests. Therefore, although it can be considered a material with light or even biocompatible cytotoxicity, it must not be considered completely harmless.
\end{abstract}

Recibido 14-11-2017 Aceptado 25-10-2018

\section{INTRODUCCIÓN}

La nanotecnología consiste en la manipulación de materiales con dimensiones que van de 1 a 100 nanómetros (nm) (1). A medida que las partículas son más pequeñas, su área de superficie se vuelve exponencialmente más grande y, por lo tanto, las NPs son más reactivas (2).

Debido a su afinidad al oxígeno y otros elementos, el titanio (Ti) no existe en su forma metálica en la naturaleza. El dióxido de titanio $\left(\mathrm{TiO}_{2}\right)$ es un compuesto mineral que se encuentra en tres diferentes estructuras cristalinas polimórficas: anatasa, rutilo y brookita. Se ha reportado que la anatasa (tamaño promedio $=3-5 \mathrm{~nm}$; dosis $=100 \mathrm{mg}$ / $\mathrm{mL}$ ) es químicamente más reactiva y genera seis veces más especies reactivas de oxígeno [por sus siglas en inglés, reactive oxyǵen species (ROS)] que el rutilio después de su irradiación con luz UV, por lo que la fase anatasa podría poseer mayor potencial citotóxico (9). Sin embargó, las ROS generadas por dicha fase no se producen bajo condiciones de luz visible $(3,4)$. 
Debido a su coloración, las nanopartículas (NPs) de $\mathrm{TiO}_{2}$ se han utilizado en piǵmentos farmacéuticos, cosméticos, productos para el cuidado de la piel y para el cuidado personal tales como protectores solares y pastas dentales, esto debido también a su capacidad para atravesar barreras biológicas. Los protectores solares y pastas dentales contienen del $1 \%$ al $10 \%$ aunque existen productos con concentraciones por debajo de $0,01 \mathrm{mg} /$ $\mathrm{mL}$ (5). En el campo de la medicina, se ha reportado el uso de diversas NPs como acarreadoras para liberación de fármacos (6), para tratamiento del cáncer (7), detección (8), diagnóstico (9) y otros tratamientos terapéuticos (10). Específicamente, las $\mathrm{NPs} \mathrm{TiO}_{2}$ se han utilizado como un potencial agente fotosensibilizador en terapia fotodinámica (11) y como componente de las partes articulares de prótesis, especialmente para cadera y rodilla (12); sin embargó, dichas prótesis, en ocasiones fracasan debido a la degradación de los materiales o a la respuesta inflamatoria crónica en el tejido que las rodea (13).

Por otro lado, se está investigando su uso como preparados de NPs de $\mathrm{TiO}_{2}$ en varios productos del cuidado de la piel para tratar acné vulgaris, dermatitis atópica, lesiones hiperpigmentadas de la piel, condiloma acuminado recurrente, entre otras (14). Asimismo, las NPs de $\mathrm{TiO}_{2}$ también parecen mostrar actividades antibacteriales bajo radiación ultravioleta (15), ampliando así su aplicación en este campo. Tradicionalmente, las partículas de $\mathrm{TiO}_{2}$ se han considerado como inertes, debido a esto, se han utilizado como "control negativo" en muchos estudios toxicológicos de partículas tanto in vitro como in vivo. Actualmente, esta visión ha cambiado tras observar el desarrollo de tumores pulmonares cancerígenos en ratas después de dos años de exposición a concentraciones altas de partículas finas de $\mathrm{TiO}_{2}$ (16). Por lo que la Agencia Internacional para la Investigación sobre el Cáncer [por sus siglas en inglés, International Agency for Research on Cancer (IARC)], ha clasificado al $\mathrm{TiO}_{2}$ dentro del grupo $2 \mathrm{~B}$ carcinó- geno (posible carcinóǵeno para humanos) (17). Un aspecto que debe tomarse en consideración, es que las NPs poseen un tamaño diminuto, el cual facilita su incorporación a las células por vía de endocitosis (18) y una vez que las NPs han traspasado la membrana, pueden afectar las funciones celulares e inducir la muerte. Existe cierta controversia sobre la biocompatibilidad de dichas NPs; algunos estudios muestran que la inhalación e ingesta de estas es segura sin causar efectos adversos (19), mientras que otros reportes señalan que incrementan el riesgo a desarrollar asma en las crías de ratones expuestos a inhalarlas (20). Además, se ha encontrado que causan efectos citotóxicos en contacto con tejido cardiaco (21) y hemaǵlutinación y hemólisis celular en cultivo con eritrocitos (22). En cambio, algunos estudios en otro tipo de líneas celulares reportan que el contacto con células epiteliales alveolares (8A549), células epiteliales bronquiales (16HBE), monocitos/macrófagos (THP-1), y macrófagos alveolares de ratón no producen citoxicidad, ni reducción del número de células viables (23). Por otro lado, las NPs, en dosis mayores a $1 \mathrm{mg} / \mathrm{L}$ muestran citotoxicidad por acción pro-oxidante en contacto de fibroblastos de ratón y de humano (24). Las condiciones para la evaluación nanotoxicológica in vitro pueden verse en la Tabla I.

En odontología, la utilización de las NPs es un campo potencial para el desarrollo de nuevos materiales de restauración, implantes dentales, irrigación de conductos, entre otras, debido a sus propiedades antibacteriales y terapéuticas (25)including dental practice with the development of silver nanoparticles (Ag NPs). Recientemente, diversos materiales dentales han incluido NPs en su composición. Por ejemplo, se han incorporado NPs de $\mathrm{TiO}_{2}$ a cementos de ionómero de vidrio, las cuales incrementan significativamente su resistencia a la flexión y compresión así como su microdureza y su efecto antibacterial, además de no interferir con la adhesión de estos con los tejidos dentales (26). 
TABLA I

CONDICIONES PARA LA EVALUACIÓN NANOTOXICOLÓGICA IN VITRO

\begin{tabular}{|c|c|}
\hline Condición & Características \\
\hline Dosis inoculada y dosis celular & $\begin{array}{l}\text { La dosis inoculada es variable y es por ello que se debe considerar } \\
\text { también, efecto de dosis-respuesta, la dosis intracelular así } \\
\text { como la densidad celular. }\end{array}$ \\
\hline $\begin{array}{l}\text { La dosis celular inoculada } \\
\text { no es siempre lo más relevante }\end{array}$ & $\begin{array}{l}\text { La dosis de NPs que se inoculan a las células no es tan } \\
\text { importante, lo que si se bene considerar es la dosis intracelular } \\
\text { por la incorporación de las NPs a las células. }\end{array}$ \\
\hline ¿Dispersas o no? & $\begin{array}{l}\text { La mayoría de las investigaciones toman precaución en tratar } \\
\text { de inocular las NPs dispersas con ayuda de agentes químicos } \\
\text { o por agitación ultrasónica, sin embargo de las NPs son } \\
\text { de naturaleza agregada en el aire, agua o alimentos, por lo } \\
\text { cual se consideran menos citotóxicas. Los aǵentes químicos } \\
\text { y la aǵitación usados para evitar la agregación de las NPs en } \\
\text { ocasiones pueden producir resultados diferentes. Además, se } \\
\text { ha demostrado que cuando se inoculación las NPs en el medio } \\
\text { de cultivo, estás se agreǵan casi de forma instantánea. }\end{array}$ \\
\hline $\begin{array}{l}\text { ¿Qué unidad de medición } \\
\text { se debe utilizar para expresar } \\
\text { la dosis? }\end{array}$ & $\begin{array}{l}\text { Existe mucha controversia respecto a la unidad de medición en } \\
\text { que se debe expresar la dosis. La mayoría de las investigaciones } \\
\text { representan mediante dosis gravimétricas ( } \mu \mathrm{g} / \mathrm{mL} \text { de medio } \\
\text { de cultivo, } \mu \mathrm{g} / \mathrm{cm}^{2} \text { del plato de cultivo, } \mu \mathrm{g} / 10^{6} \text { células) y, en } \\
\text { ocasiones, se utiliza la molaridad por ml de medio de cultivo } \\
(\mathrm{M} / \mathrm{mL}) \text {. Las concentraciones dependerán de la naturaleza de } \\
\text { las NPs y si se encuentran suspendidas en un vehículo. }\end{array}$ \\
\hline $\begin{array}{c}\text { Las células blaneo } \\
\text { a utilizar }\end{array}$ & $\begin{array}{l}\text { Es de vital importancia tomar en cuenta el tipo de célula } \\
\text { blanco a evaluar y la cantidad de divisiones celulares (PDL, de } \\
\text { inǵlés population doubling level) que se han efectuado en las } \\
\text { células a evaluar. }\end{array}$ \\
\hline
\end{tabular}

Por lo antes mencionado, el presente artículo tiene como propósito analizar el impacto biológico de las NPs de $\mathrm{TiO}_{2}$ para determinar su citotoxicidad, producción de ROS así como inducción de estrés oxidativo, a través de una revisión sistemática de la bibliografía publicada hasta el momento sobre la biocompatibilidad de éstas en contacto con células orales. Adicionalmente, se incluyó una breve sección que brinda un panorama general de los métodos de síntesis de NPs de $\mathrm{TiO}_{2}$.

\section{METODOLOGÍA}

En la presente revisión sistemática, los datos disponibles sobre las NPs de $\mathrm{TiO}_{2}$ fueron recopilados de la base de datos electrónica PubMed y Science Direct, de acuerdo con recomendaciones PRISMA para revisiones sistemáticas (27), durante el mes de octubre-noviembre de 2017. Se realizó una búsqueda de los métodos de síntesis de NPs de $\mathrm{TiO}_{2}$ con el fin de enriquecer el contenido de la revisión, así como ofrecer un contexto adecuado del tema. La búsqueda electrónica se realizó en dichas bases de datos con las siguientes palabras clave y términos MeSH: viability AND oral cells AND titanium dioxide nanoparticles; Cytotoxicity AND titanium dioxide nanoparticles AND oral cells; Prostaglandin $\mathrm{E}_{2}$ AND titanium dioxide nanoparticles AND oral cells; Oral cells AND oxidative stress AND titanium dioxide nanoparticles; 
Reactive oxygen species generation AND titanium dioxide nanoparticles AND oral cells.

Para refinar aún más la búsqueda, se tomaron los siguientes criterios de inclusión: Artículos de texto completo en idioma inglés centrados en el objetivo de esta revisión, que son los aquellos estudios originales publicados en revistas sobre biocompatibilidad de las NPs de $\mathrm{TiO}_{2}$ en contacto con células orales. La evaluación de la calidad de los artículos fue determinada por la investigación original y metodológicamente coherente y publicada en revistas indizadas de revisión por pares.
Las referencias bibliográficas de los artículos consultados también se consideraron como búsqueda manual, como artículos pertinentes no incluidos en la búsqueda electrónica si contaban con los criterios de inclusión de la búsqueda. La extracción de datos, la recolección, manejo y análisis de datos consistió en la descripción de la evidencia relevante, la cual se presenta en diagrama de flujo de acuerdo a PRISMA (Fig. 1). Además, se presenta una evaluación crítica de los resultados de forma ordenada bajo encabezados apropiados para cada uno de ellos.

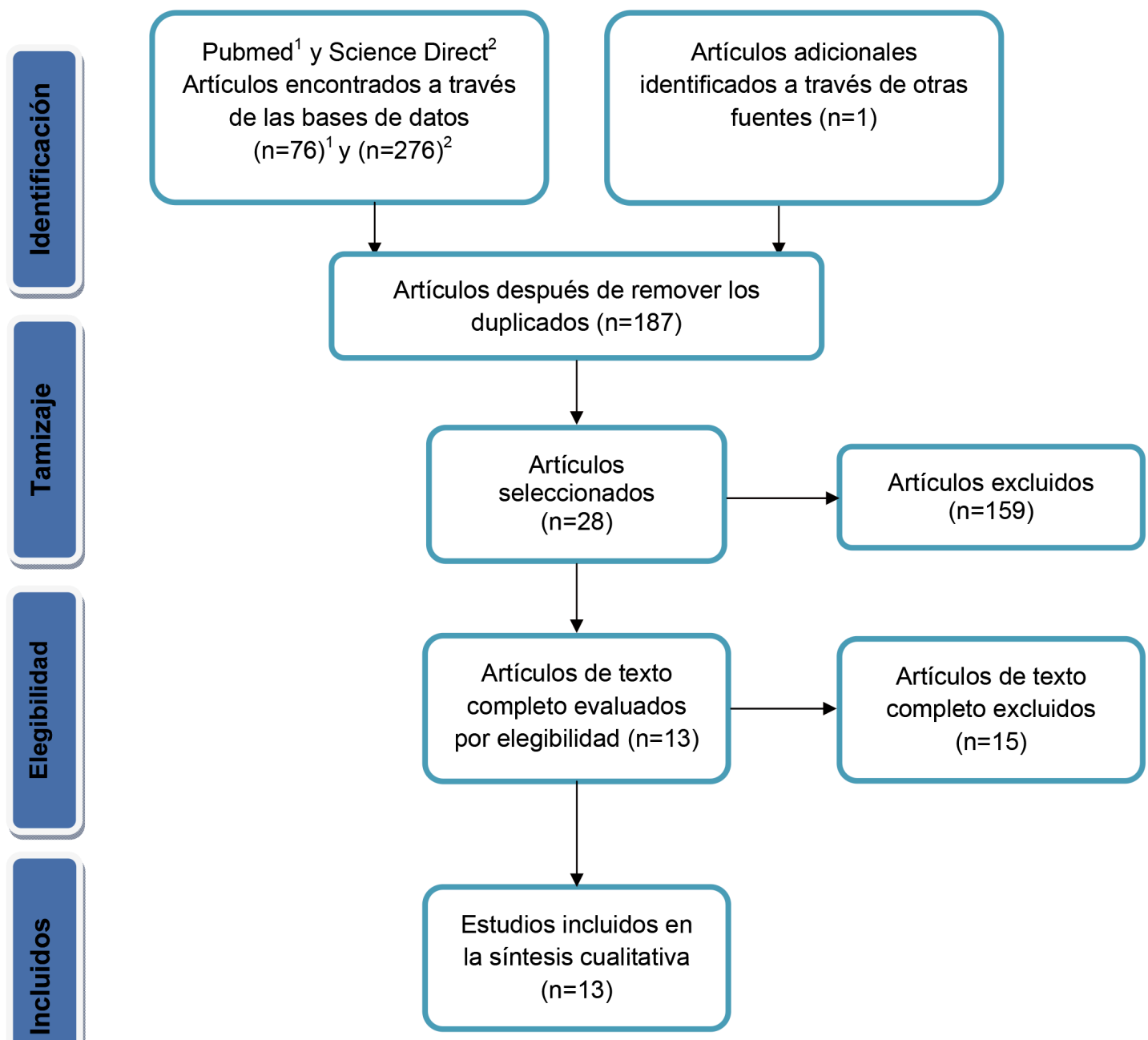

Fig. 1. Diagrama de flujo PRISMA de la revisión sistemática. 


\section{DESARROLLO DE LA REVISIÓN SISTEMÁTICA}

\section{Generalidades de los métodos de síntesis de NPs de $\mathrm{TiO}_{2}$}

Uno de los aspectos importantes que se debe de considerar cuando se pretende utilizar un material estructurado es el método de síntesis, ya que este va encaminado a controlar la morfología, tamaño y el ambiente químico en la superficie de la NPs. En la literatura, desde hace más de dos décadas, existen reportes de síntesis de $\mathrm{TiO}_{2}$, enfocados a la preparación de polvos, cristales, películas delgadas y estructuras. Existe una gran gama de métodos de síntesis que pueden ser utilizados para obtener NPs de $\mathrm{TiO}_{2}$; desde los físicos hasta los biológicos, por lo que dependiendo de la aplicación final, será el método utilizado para elaborarlas (28).

Cuando se desea tener un buen control de la estequiometría, el tamaño y la forma los métodos más utilizados son los métodos químicos, entre los que podemos mencionar sol-gel, micro-emulsión, hidrotermal o deposición química de vapor. Sin embargoo, estos métodos presentan algunas desventajas tales como el uso de precursores caros, tiempos largoos de síntesis y, en algunos casos, presencia de impurezas.

El método de sol-gel, es en base húmeda; en la que se realiza una reacción de hidrólisis del alcóxido o haluro del metal precursor, dando paso a la condensación de la fase inorgánica; siendo uno de los más utilizados. Uno de los grupos de investigación que estudiaron algunas de las variables que interviene en este proceso es el de Sugimoto y col. (29-33); ellos variaron la relación del precursor con un grupo amino, $\mathrm{pH}$, temperatura y tiempo de reacción y encontraron que controlando estas variables podían obtener NPs con diferentes morfologías y tamaños. Su y col. (34), sintetizaron $\mathrm{NPs}^{\mathrm{de}} \mathrm{TiO}_{2}$ a partir de la hidrólisis de n-Butóxido de Titanio (IV) en isopropanol con un tamaño variable de 4-35nm con morfologías elipsoidales y polígonales. Wetchakun y Phanichphant (35), utilizaron etanol absoluto para llevar a cabo la hidrólisis y una membrana de celofán para aumentar el proceso de difusión, reportando NPs de $\mathrm{TiO}_{2}$ con alta pureza y tamaños de 15-60nm.

Otro de los métodos, en el cual utiliza agua como solvente es el de micro-emulsión donde se forman micelas para controlar el tamaño y la morfología de las NPs de $\mathrm{TiO}_{2}$ (36-42). En este método las variables que se deben de controlar son la solubilización, tensión superficial, conductividad y difusión. Kim y cols. (36) sintetizaron $\mathrm{NPs}_{\text {de }} \mathrm{TiO}_{2}$, en agua y en amoniaco y obtuvieron partículas amorfas con tamaños de 10-20nm; al calcinar a $600^{\circ} \mathrm{C}$ obtuvieron la fase anatasa y a $900^{\circ} \mathrm{C}$ la fase rutilo. Li y col. (40) utilizaron una fase oleosa y un surfactante no iónico y obtuvieron NPs amorfas; la fase anatasa se obtuvo utilizando rangos de temperatura de $200-750^{\circ} \mathrm{C}$ y la fase rutilo con una temperatura mayor a $750^{\circ} \mathrm{C}$. Zhang y col. (11) sintetizaron $\mathrm{TiO}_{2}$ cristalino, utilizando ácidos y temperatura ambiente; su aporte principal fue la relación de la acidez con la relación de cristalinidad de la nanopartícula.

También, diferentes grupos de investigación (16-23), han sintetizado NPs de $\mathrm{TiO}_{2}$ utilizando método hidrotermal o solvotermal. Nian y col. (23) prepararon barras de anatasa en ausencia de surfactantes controlando el pH de la reacción.

En definitiva, se han reportado una gran variedad de métodos de síntesis para fabricación de NPs de $\mathrm{TiO}_{2}$ con diferentes morfologías y tamaños. De ellos, el método de sol-gel es el más recomendado para sintetizar nanopartículas monodispersas, que es un factor importante para obtener los beneficios de los materiales a escala nanométrica y que dichas NPs puedan ser utilizadas en aplicaciones biológicas.

Es importante mencionar que se debe hacer un estudio detallado de la toxicidad y la biocompatibilidad de estas NPs, ya que en la literatura se muestra principalmente los estudios enfocados a las aplicaciones de $\mathrm{TiO}_{2}$. En lo que se refiere a su biocompati- 
bilidad, se han realizado trabajos de investigación acerca de la citotoxicidad de las NPs de $\mathrm{TiO}_{2}$ sobre diversas células de animales y de humanos, e incluso es probable que sea uno de los nanomateriales más estudiados; sin embargo, existen pocos reportes acerca de la biocompatilibidad en células orales.

\section{Citotoxicidad de las NPs de $\mathrm{TiO}_{2}$ en cultivo de células orales}

Al llevar a cabo la revisión sistemática, uno de los aspectos que se observan en los estudios publicados hasta el momento es la imposibilidad de realizar una comparación cuantitativa debido a la gran variabilidad en las condiciones entre los diversos estudios. Así, se emplean diversos métodos de síntesis que generan diferentes tamaños de partícula y de morfología, las dosis de las NPs difieren entre sí, así como el número de subdivisiones celulares de la población, el tipo de células, ya sea obtenidas a través explantes de tejido o adquiridas como células inmortalizadas ATCC y los cultivos celulares primarios empleadas en los experimentos. Debido a esto, la siguiente revisión sistemática está enfocada en un análisis cualitativo de los datos publicados en la literatura hasta el momento.

En las investigaciones que han sido publicadas sobre la citotoxicidad de las NPs de $\mathrm{TiO}_{2}$ en contacto con células orales (vea Tabla II), la viabilidad celular se ha evaluado por dos métodos, el ensayo de bromuro de 3-(4,5-dimetiltiazol-2-ilo)-2,5-difeniltetrazol (MTT) y el tetrazolio soluble en agua (WST1). Ambos métodos son muy eficientes e igualmente válidos para terminar la citotoxidad de un material; sin embargo, el WST-1 es una prueba más sensible capaz de detectar actividad celular aun cuando el conteo es escaso.

En un estudio, en el cual se emplearon NPs de $\mathrm{TiO}_{2}$ dopadas y sin dopar con nitrógeno a través del método del sol-gel, se observaron agregados de partículas entre 100 a $300 \mathrm{~nm}$, conformados por NPs primarias de 20-30 nm, en ambos casos. En el análisis de Difracción de Rayos X (DRX), se observó que el dopaje con nitrógeno dio como resultado la formación de $\mathrm{TiO}_{2}$ principalmente en fase rutilo, mientras que el $\mathrm{TiO}_{2}$ sin dopar fue principalmente anatasa. Se registró la actividad mitocondrial de las células epiteliales gingivales humanas primarias (hGEPs), en contacto con NPs de $\mathrm{TiO}_{2}$ con y sin ser dopadas con nitrógeno, con o sin luz azul (485 nm de longitud de onda) usando WST-1 (41). Además, realizaron un ensayo de especies reactivas de oxígeno (ROS) intracelular, el cual mostró que las partículas causaban una respuesta de estrés en hGEPs cuando se exponían a 1 hora de luz azul, aunque esto no dio como resultado una liberación detectable de citoquinas. Los resultados muestran que las NPs de $\mathrm{TiO}_{2}$ dopado con nitrógeno tienen actividad antibacteriana cuando se exponen a la luz azul y son biocompatibles a estas concentraciones. El tratamiento con NPs de $\mathrm{TiO}_{2}$ dopadas con nitrógeno expuestas a luz ambiental resultó en un aumento ligero, aunque no significativo, de la proliferación celular. Además, el metabolismo celular fue significativamente elevado en las células expuestas a $50 \mathrm{y} 100 \mu \mathrm{g} / \mathrm{mL}$ durante 24 horas, independientemente del tratamiento con luz. Al parecer, dichas NPs estimularon el metabolismo celular a concentraciones bajas e intermedias, pero no fueron citotóxicas en ninguna concentración probada. Debido a resultados de investigaciones como las señaladas anteriormente, acerca de las NPs y tubos de $\mathrm{TiO}_{2}$ y de otras llevadas a cabo con otras líneas celulares no provenientes de la cavidad oral, se considera por varios grupos de investigación, como un agente que potencialmente puede estimular la proliferación celular por lo que se ha propuesto como recubrimiento para implantes dentales, sin embargo aunque la adición de NPs de $\mathrm{TiO}_{2}$ ha mejorado la frecuencia de éxito de los implantes en experimentos in vivo, todavía dicha tasa de éxito no es completamente satisfactoria (42).

De igual manera, en otro estudio se utilizaron células epiteliales bucales humanas (TR146), las células se pusieron en contac- 


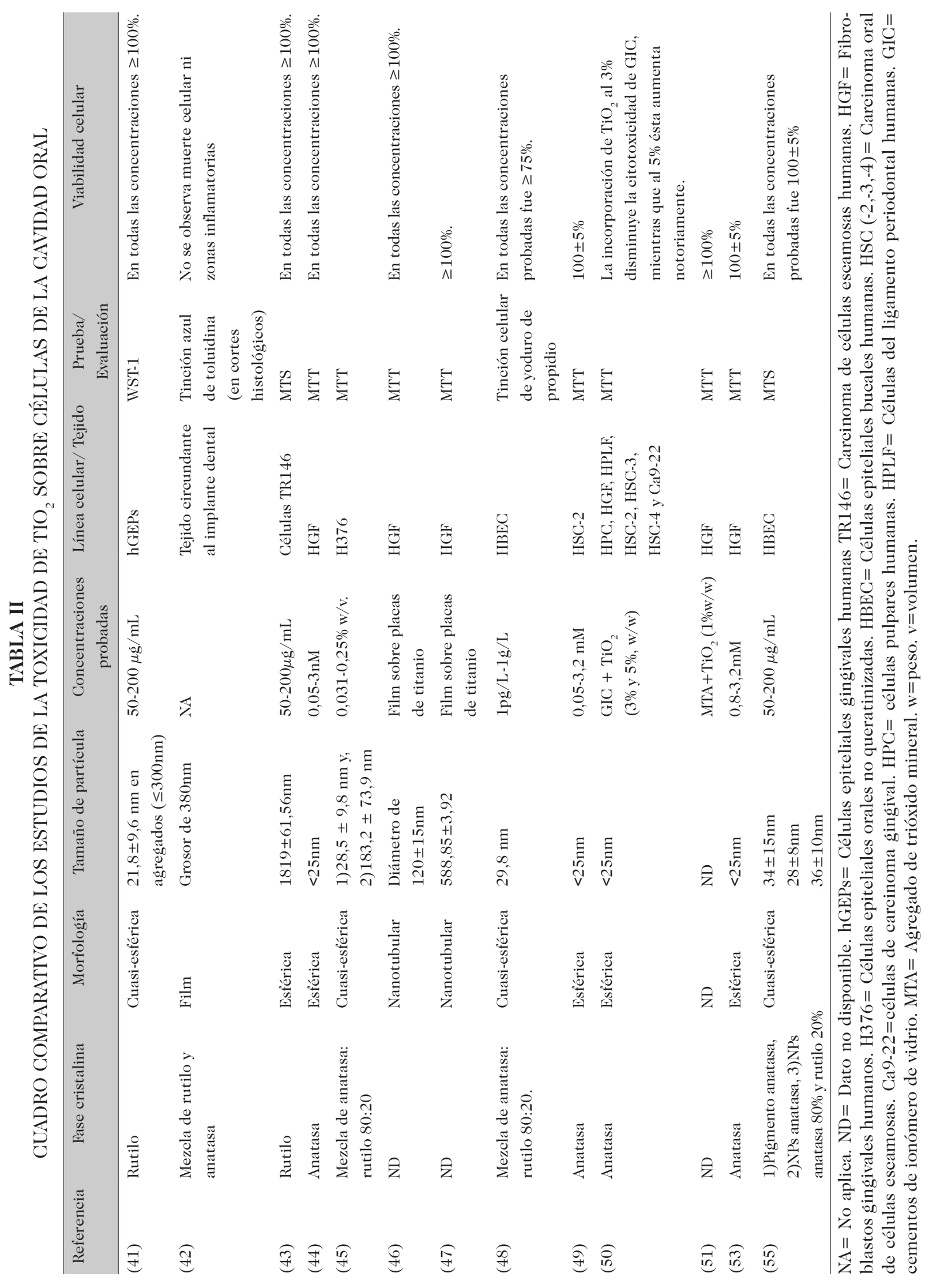


to con dosis entre $1-200 \mu \mathrm{g} / \mathrm{mL}$ de NPs de $\mathrm{TiO}_{2}$ hidrófobas-rutilo (tamaño promedio de $20 \mathrm{~nm}$, recubiertas con dimeticona) y NPs de $\mathrm{TiO}_{2}$ hidrófilas-rutilo (tamaño promedio $20 \mathrm{~nm}$, recubiertas con glicerol). En sus resultados indicaron que la actividad mitocondrial de las células puestas en contacto con cada uno de los tipos de NPs fue mayor en comparación con el control celular. En este estudio se realizó una prueba de mutaǵenicidad (ensayo de mutación del gen HPRT), y se encontró que ninguna de las NPs desencadenó la mutación génica en las células epiteliales bucales. En dicho estudio, las NPs hidrofílicas produjeron aǵlomerados de casi el doble de tamaño que las NPs hidrofóbicas de $\mathrm{TiO}_{2}$ al ser suspendidas tanto en PBS como en saliva artificial. Una posible explicación para la aparente proliferación celular podría ser que dicho incremento es detectado en el ensayo como consecuencia del aumento de la actividad metabólica mitocondrial, la cual podría implicar una situación de estrés celular. Para aclarar este problema, se examinó el potencial de membrana mitocondrial, el cual es indispensable para mantener la función fisiológica de las células. Una pérdida en el potencial de membrana produce lo que genera agotamiento de la energía y muerte celular. El colorante TMRM funciona como un sensor de potencial de membrana mitocondrial y se usó para evaluar los cambios temporales. El efecto desequilibrante del potencial de membrana mitocondrial fue más pronunciado para las partículas hidrofílicas que para las hidrofóbicas. Por tanto, un potencial de membrana reducido de las mitocondrias indica el primer signo de muerte celular programada. La producción de especies reactivas de oxígeno (ROS) se evaluó en ausencia de luz UV. Los datos revelaron que las partículas hidrófobas causaron una mayor producción de ROS en comparación con las células no tratadas. Sin embargó, la fluorescencia relativa obtenida fue marcadamente menor que el control positivo (43). Esto puede interpretarse como que existe una generación de ROS por parte de estas partículas, que pueden producir cambios en las funciones mitocondriales que son seguidos por alteraciones celulares que desencadenan el inicio del proceso apoptótico.

Por otra parte, se ha reportado que el contacto de NPs de $\mathrm{TiO}_{2}$, a diferentes concentraciones, con contacto fibroblastos gingivales humanos [del inǵlés Human Gingival Fibroblast (HGF)], no mostraron citotoxicidad. Además, se observó proliferación celular a bajas concentraciones y una disminución en el número de células viables a mayor concentración; es decir que sigue un patrón dosis-dependiente (44).

En un estudio en el que se utilizaron NPs de $\mathrm{TiO}_{2}$ en una mezcla de anatasa-rutilo (en relación 80:20, estabilizadas en solución acuosa, Sigma-Aldrich) comparadas con micropartículas de $\mathrm{TiO}_{2}$ se encontró que el $\mathrm{TiO}_{2}$, tanto nano como micrométrico, fue bien tolerado por las células al emplear un modelo de células epiteliales orales no queratinizadas (H376) a concentraciones que van de 0,031\% a $0,250 \%$ peso/volumen. En dicho estudio se observó, a través de la medición de la liberación de lactato deshidrogenasa $(\mathrm{LDH})$, la disrupción de la membrana celular inducida por las NPs de $\mathrm{TiO}_{2}$; la liberación de LHD para todas las concentraciones de $\mathrm{TiO}_{2}$ tanto nano como micrométrico, fue menor al 25\%. Esto indicaría que, en dicho porcentaje de células, se libera una enzima citosólica estable en etapas tempranas durante la necrosis y en etapas tardías durante la apoptosis (45). Esto se interpreta como una citotoxicidad ligera, sin encontrar diferencias en dicha toxicidad atribuibles al tamaño de partícula.

En un estudio acerca de la citotoxicidad de nanotubos de $\mathrm{TiO}_{2}$ (diámetro $=120$ $\mathrm{nm}$ ) en HGF monitorizados por la actividad de la deshidrogenasa mitocondrial (ensayo de MTT), después de un período de exposición de 72 h, se observó un ligero aumento en el número de células metabólicamente activas (46), lo cual clínicamente se traduciría como proliferación celular.

Otro aspecto que es abordado es la posibilidad de emplear nanotubos de $\mathrm{TiO}_{2}$ como 
reservorio de lenta liberación de factores de crecimiento tales como el Factor de crecimiento fibroblástico 2. En este estudio se muestra una citotoxicidad de los nanotubos de $\mathrm{TiO}_{2}$ en forma de film sobre placas de $\mathrm{Ti}$ que podría ser empleado para fines prácticos como un recubrimiento para implantes. $\mathrm{Al}$ evaluar la viabilidad celular de los HGF se encontró una viabilidad ligeramente mayor que el control, la cual aumentaba aún más si se exponía a diferentes concentraciones del Factor de crecimiento fibroblástico 2 (FGF2) de manera dosis dependiente, lo cual a su vez también incrementa la adhesión celular a las placas de Ti funcionalizadas con los nanotubos y el FGF2. En este estudio se remarca la importancia de continuar estudiando la posibilidad de emplear dicho NPs de $\mathrm{TiO}_{2}$ para liberación prolongada de factores de crecimiento de manera local, lo cual abriría la posibilidad de generar otras líneas de investigación prometedoras (47).

Además, en consistencia con esta investigación, se probaron las NPs de $\mathrm{TiO}_{2}$ (pure$\mathrm{za} \geq 99,5 \%$; anatasa $/$ rutilo $=80: 20 ; \mathrm{y}$ tamaño de partícula $=27 \mathrm{~nm}$ ) a concentraciones desde $100 \mathrm{mg} / \mathrm{L}$ en diluciones seriadas hasta $1 \mathrm{pg} / \mathrm{L}$ frente a las células TR146. Las células tratadas fueron igual de viables (más del 98\% de viabilidad relativa) que el grupo control no tratado. Dichas células tratadas son iǵual de viables (más del 98\% de viabilidad relativa) que el grupo control no tratado. En las células TR146 con NPs de $\mathrm{TiO}_{2}$, la muerte celular significativa sólo se detectó a una dosis de $1 \mathrm{~g} / \mathrm{L}$ con una disminución del 24\% en la viabilidad celular relativa.

Otro aspecto de discusión en este estudio, es cuando las NPs de $\mathrm{TiO}_{2}$ están en suspensión en cuyo caso tienden a agregarse y el grado de agregación depende del dispersante utilizado. Esto se demostró al comparar el tamaño hidrodinámico de las NPs suspendidas en agua ultrapura, DMEM/F-12 y únicamente DMEM. Los resultados mostraron que el tamaño hidrodinámico de las NPs en DMEM fue 33-37\% menor que el tamaño de las partículas suspendidas en agua ultra- pura; en cambio, en DMEM/F-12 el tamaño fue un poco mayor que en aquellas partículas suspendidas en DMEM pero $28 \%$ más pequeñas que las suspendidas en agua ultrapura. Los valores absolutos del potencial zeta se desplazaron hacia potenciales negativos en los medios de cultivo, DMEM/F-12 (7,6 $\mathrm{mV})$ y DMEM $(-11,6 \mathrm{mV})$ en comparación con los valores del potencial zeta del agua ultrapura $(+21,8 \mathrm{mV})$. Al ser suspendidas en aǵua las NPs de $\mathrm{TiO}_{2}$ con un tamaño de 29,8 nm incrementó el tamaño a 392,2 $\mathrm{nm}$ en aǵua ultrapura cuyo $\mathrm{pH}$ fue de 6,8 , DMEM/F-12 con un pH de 7,4 con $282 \mathrm{~nm}$ y DMEM completo ( $\mathrm{pH} 7,4)$ con $260,9 \mathrm{~nm}$, por lo que el medio es un factor determinante para la aǵregación de las partículas. Además, al parecer, existe una relación directa entre el $\mathrm{pH}$ del medio de cultivo, el potencial zeta y la aglomeración de las NPs; esto es un aspecto importante ya que de dichas condiciones dependerá el tamaño final de los agregados de $\mathrm{TiO}_{2}$. $\mathrm{Al}$ observar los resultados de estudios sobre vialidad celular del contacto de las NPs de $\mathrm{TiO}_{2}$ en cultivo celular, éstas tienden a agregarse entre sí muy fácilmente al entrar en contacto con el medio de cultivo celular, formando aglomerados de NPs (Fig. 2), que muestran menor toxicidad los aglomerados cuando de compara con las NPs dispersas. Al parecer, la transición entre la agregación y dispersión de estas NPs produce resultados experimentales diferentes dependiendo la naturaleza de dichos experimentos. Dado los datos de los reportes anteriores, la citotoxicidad de dichas NPs se genera en función de la concentración; por lo que mientras las concentraciones sean bajas, la citotoxicidad del material es mínima. En el estudio referido se observó una baja citotoxicidad en TR146. En dichas células, la muerte celular sólo se detectó a una dosis de $1 \mathrm{~g} / \mathrm{L}$, con una disminución del 24\% en la viabilidad celular (48).

Por otra parte, es muy escasa la información sobre el efecto de las $\mathrm{NPs}$ de $\mathrm{TiO}_{2}$ sobre las células cancerígenas. Debido a que estas células exhiben una alta capacidad de proli- 


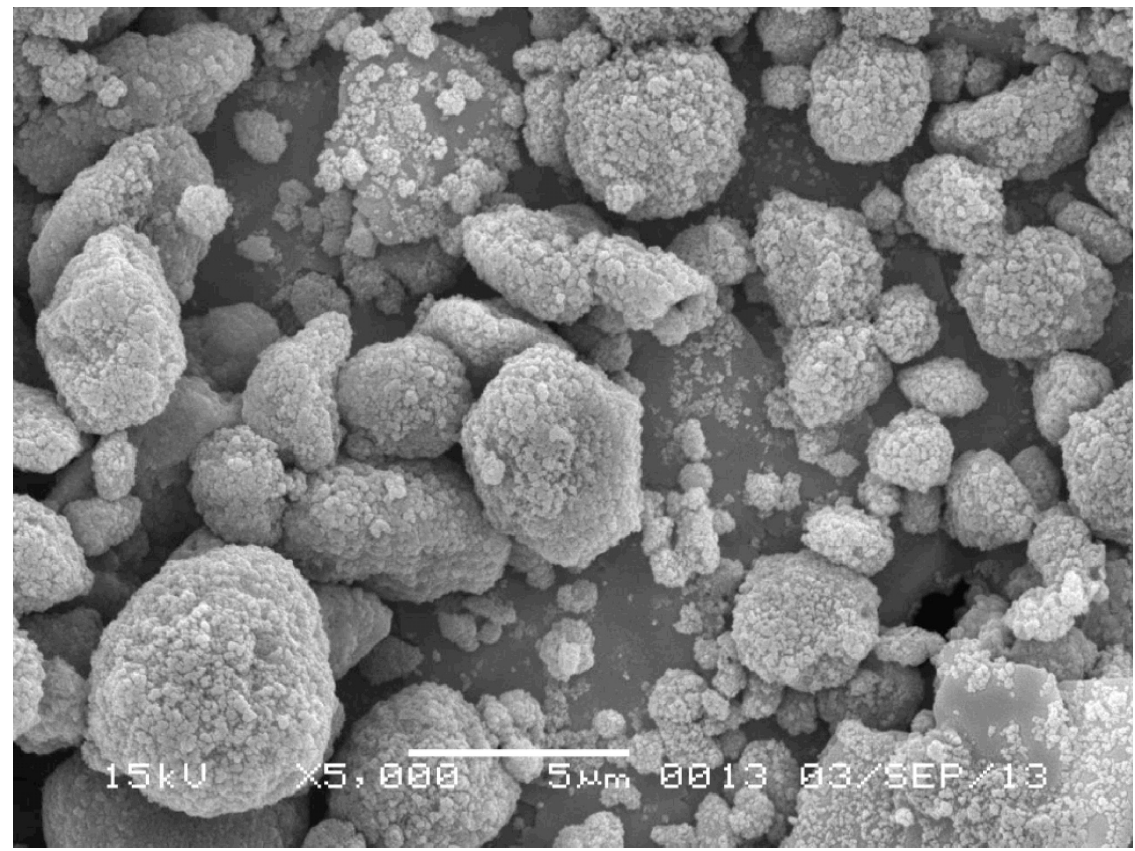

Fig. 2. Imagen representativa de la ultra estructura de las nanopartículas (NPs) de dióxido de titanio $\left(\mathrm{TiO}_{2}\right)$. Las NPs de $\mathrm{TiO}_{2}$ fueron disueltas en agua bidestilada y agitadas en ultrasonido durante 5 min antes de inocularse en el medio de cultivo. Una gota de medio de cultivo conteniendo las NPs de $\mathrm{TiO}_{2}$ fue colocada sobre un portamuestras de aluminio; el espécimen se dejó secar durante 48 hrs a temperatura ambiente. La muestra fue recubierta con una capa de oro de $50 \mathrm{~nm}$. La topografía de las NPs de $\mathrm{TiO}_{2}$ fue observada en microscopia electrónica de barrido (SEM, del inglés Scanning electron microscope, JSM-6360LV; JEOL, Tachikawa, Japón) con electrones secundarios $(\mathrm{x} 10,000$ y $15 \mathrm{kV})$.Se observa una agregación de las nanopartículas (NPs) de dióxido de titanio $\left(\mathrm{TiO}_{2}\right)$.

feración y, tomando en cuenta el comportamiento reportado en células normales de las NPs de $\mathrm{TiO}_{2}$ se podría suponer que tampoco serian citotóxicas para las células cancerígenas. Para demostrar esto, se pusieron en contacto NPs de $\mathrm{TiO}_{2}$ con células humanas de carcinoma escamoso [del inglés Human Squamous Carcinoma (HSC-2)], las cuales no mostraron citotoxicidad y al combinarse con agentes quimioterapéuticos tampoco se potencializó el efecto antitumoral de dichos fármacos [doxorubicina, melfalán, 5-fluorouracilo (5-FU), gefitinib, y docetaxel] (49), sin mostrar diferencias estadísticamente significativas.

Otra línea de estudios involucra la incorporación de las NPs de $\mathrm{TiO}_{2}$ a materiales de uso odontológico en porcentajes menores al 5\%, ya que dopar un material con un por- centaje mayor de NPs de cualquier composición (no necesariamente de $\mathrm{TiO}_{2}$ ) genera un decremento de las propiedades físicas originales del material. En este sentido, se realizó un estudio, en el cual se incorporaron NPs de $\mathrm{TiO}_{2}(3$ y 5\%) en diferentes cementos de ionómero de vidrio (GIC, de 0 a $40 \mathrm{mg} / \mathrm{mL}$ ). Se encontró una citotoxicidad moderada, independientemente de la composición del GIC y la presencia o la ausencia de NPs de $\mathrm{TiO}_{2}$; sin embargoo, extrañamente, las células de cáncer orales fueron más sensibles que las células orales normales (50). También se realizó un estudio para evaluar la citotoxicidad del MTA y MTA con NPs de $\mathrm{TiO}_{2}$ al $1 \%$ en peso durante 24 y $48 \mathrm{~h}$ después de ponerse en contacto con HGF. Los resultados mostraron un aumento en la viabilidad celular de ambos grupos, y en ambos intervalos de 
tiempo, respecto al control de células sin inocular. Sin embargo, una de las deficiencias que impiden la reproducibilidad de este estudio, es que no se especifica el tamaño de partícula ni el método de obtención de las nanopartículas (51).

$\mathrm{Al}$ realizar los ensayos de viabilidad celular, una consideración importante es que el método del MTT o incluso el WST-1 pueden dar resultados erróneos. El primero, por ejemplo, se basa en la reducción metabólica del MTT realizada por la enzima mitocondrial succinato-deshidrogenasa en un compuesto coloreado de color azul-violeta (formazán), permitiendo determinar la función mitocondrial de las células tratadas. En el empleo de este ensayo se pueden producir lecturas erróneas debido a que ciertas NPs reaccionan con los reactivos empleados en los ensayos de citotoxicidad al incorporar un solvente orgánico aprótico, comúnmente dimetil sulfóxido, lo que genera la interpretación de las partículas como células vivas o con actividad mitocondrial en el ensayo (52). Para evitar que esto ocurra debe trasportarse el sobrenadante de los platos de cultivo a una microplaca nueva antes de la lectura de la densidad óptica. Esto resuelve el problema dando una lectura precisa de la cantidad de actividad metabólica que se entiende como el número de células viables en el cultivo.

\section{Acción pro-inflamatoria de las $\mathrm{NPs}_{\text {de }} \mathrm{TiO}_{2}$ en células orales}

A pesar de que las NPs de $\mathrm{TiO}_{2}$ no mostraron citoxicidad en cultivo con HGF, estas NPs inducen a un incremento en la producción de prostaglandina $\mathrm{E}_{2}\left(\mathrm{PGE}_{2}\right)$ con la mínima concentración de $0,2 \mathrm{mM}(47)$, y en mayor grado cuando se adiciona interleucina- $1 \beta$ (IL-1 $\beta$ ), un agente inflamatorio conocido y estudiado previamente por nuestro grupo de investigación. El contacto de las $\mathrm{NPs}$ de $\mathrm{TiO}_{2}$ con HGF, refleja una actividad pro-inflamatoria cuyo mecanismo se ha identificado por el incremento de la expresión de ciclooxigena- sa 1 y 2 al estar presente la IL-1 $\beta$, aǵravando el efecto pro-inflamatorio (53). Esta información es de suma importancia, ya que la presencia de NPs de $\mathrm{TiO}_{2}$ en cavidad bucal y ante una enfermedad inflamatoria crónica (por ejemplo en la periodontitis y ginǵivitis) agrava significativamente la inflamación, pero al conocer los mecanismos pro-inflamatorios se puede contrarrestar dicha inflamación con antiinflamatorios no esteroideos.

Es imprescindible mencionar que la acción pro-inflamatoria puede ser el resultado de la presencia de lipopolisacáridos (LPS) en las NPs; sin embargo, se ha eliminado esta posibilidad a través de la adición intencional de LPS en el cultivo, concluyendo que su presencia (a una concentración de 0,2 mM de NPs de $\mathrm{TiO}_{2}$ con 9,0 ng de LPS) no es suficiente para inducir una acción pro-inflamatoria, siendo las NPs de $\mathrm{TiO}_{2}$ la causa directa de la inducción del efecto inflamatorio en cultivo de HGF $(44,52)$.

Se encontró un único estudio, realizado por nuestro grupo de trabajo, donde se realizó un análisis con técnica metabolómica de las NPs de $\mathrm{TiO}_{2}$ en HGF inducidas a un estado inflamatorio con IL-1 $1 \beta$. Las NPs de $\mathrm{TiO}_{2}$ aumentaron significativamente la producción de prostaglandina $\mathrm{E}_{2}$ inducida por IL- $1 \beta$ y expresión de la proteína COX-1 y COX-2. IL-1 $\beta$, redujo las concentraciones intracelulares de metabolitos primarios en general, especialmente las vías sintéticas de los aminoácidos, el ciclo de urea, poliamina, S-adenosilmetiona y glutatión (52), ciclos relacionados con el proceso inflamatorio e incremento del estrés oxidativo. Por tal motivo, se recomendaría el uso cuidadoso de los materiales dentales que contienen NPs de $\mathrm{TiO}_{2}$ en pacientes con enfermedades pro-inflamatorias agudas o crónicas como ǵingivitis o periodontitis que son muy comunes en la cavidad oral.

\section{Incorporación de las $\mathrm{NPs}$ de $\mathrm{TiO}_{2}$ en las células orales}

La interacción de las NPs de $\mathrm{TiO}_{2}$ con las células es un tema de relevancia para 
comprender los mecanismos de citotoxicidad de dicho material. En un estudio, se identificó que la incorporación de las NPs de $\mathrm{TiO}_{2}$ a las células se lleva a cabo por medio de endocitosis por vía de receptores tipo toll 4 (TLR 4) (54). La observación con microscopio electrónico de transmisión demostró la incorporación de $\mathrm{NPs}$ de $\mathrm{TiO}_{2}$ en forma de endosoma temprano y la inducción de formación de vesículas lisosomales de las células (55).

En células orales normales de HGF, HPLF y cancerígenas HSC-2 se ha observado la incorporación de las NPs de $\mathrm{TiO}_{2}$. A pesar de la formación de agregados de NPs, algunas logran incorporarse a la célula y estas se depositan y coexisten como vesículas con organelos celulares donde las NPs son vacuolizadas para su eliminación. Esta atracción o incorporación dentro de las células puede ser causa de la interacción entre los grupos amino y carboxilo de la membrana celular (con carga negativa) y la superficie de las NPs de $\mathrm{TiO}_{2}$ (con carga positiva) (55). Sin embargo, el mecanismo de incorporación de las NPs de $\mathrm{TiO}_{2}$ a las células aún no se conoce ampliamente. Diversos estudios señalan que las NPs, después de incorporarse a la célula se posicionan como endosomas y se obsrva la atracción de lisosomas en el citoplasma $(44,53)$. En otro estudio con NPs de $\mathrm{TiO}_{2}$ de rutilo, hidrofílicas $(20 \mathrm{~nm})$ y de rutilo hidrofóbicas (20 nm) se observaron diferencias signnificativas en la localización de partículas intracelulares en células epiteliales bucales humanas (TR146). Las partículas hidrofílicas se encontraron libremente distribuidas en el citoplasma, mientras que sus homólogas hidrófobas fueron engullidas en estructuras vesiculares; por lo tanto, los efectos secundarios citóxicos fueron bajos ya que las partículas aisladas del citoplasma no reaccionan con las estructuras intracelulares. $\mathrm{Al}$ parecer, las partículas hidrófobas no se unieron a las vesículas; en consecuencia, pudieron interferir con la membrana mitocondrial externa, alterando el potencial de membrana mitocon- drial que es un elemento indispensable para la homeostasis celular (43).

A través de la revisión de la literatura es evidente que se han observado diferencias en la localización de NPs de $\mathrm{TiO}_{2}$ en el interior de las células. Esto puede deberse a diversos factores intrínsecos a la morfología, tamaño, método de síntesis y agente estabilizador de las NPs. Es indudable que el medio en el que dichas NPs son solubilizadas juega un papel importante en su interacción con las células dado el cambio producido en el potencial $Z$ de la partícula y la formación de aǵlomerados.

\section{PERSPECTIVAS FUTURAS}

En un futuro, es necesario contar con mayor evidencia científica para determinar la biocompatibilidad y la aplicación segura de las NPs de $\mathrm{TiO}_{2}$ en contacto con las células orales. Para esto, el estudio de perfiles metabólicos será capaz de proporcionar información sobre la toxicidad de las NPs de $\mathrm{TiO}_{2}$, los procesos patológicos asociados a éstas y su influencia en las funciones bioquímicas. Los estudios metabolómicos han sido ampliamente utilizados en la evaluación de la seguridad de los productos químicos como un método para identificar nanotoxicidad en los órganos blancos y el mecanismo toxicológíico a través de biomarcadores de orina y suero. Una técnica metabolómica selectiva es la espectroscopía de resonancia magnética nuclear (RMN), acoplado con el patrón de reconocimiento (PR). Las investigaciones futuras podrían emplear dichos métodos, ya que éstos pueden ofrecer información nanotoxicológica rápida y no invasiva, sólida y reproducible (56). De esta manera, mediante pruebas de expresión metabólica es necesario determinar las vías inflamatorias de señalización y el mecanismo de interacción de los metabolitos, así como crear estrategias terapéuticas específicas para la aplicación de antiinflamatorios como método de prevención y determinar los efectos dosis-respuesta de las NPs de $\mathrm{TiO}_{2}$ en diver- 
sos intervalos de tiempo, particularmente en estudios in vivo. La demostración de la bioseguridad en el uso de las NPs de $\mathrm{TiO}_{2}$ deben estar encaminadas a probar su biocompatibilidad en modelos animales.

Se concluye que los trabajos de investigación encaminados al estudio de la interacción de las NPs de $\mathrm{TiO}_{2}$ con células y tejidos bucales es limitada debido a que este tipo de materiales se usaban en otras áreas de aplicación, como fotocalizadores o portadores de carǵa por mencionar algunos. Las publicaciones están orientados a pruebas de citotoxicidad en otro tipo de células somáticas (no provenientes de la cavidad oral) y órganos que pueden ser afectados durante los métodos de síntesis o manipulación de la NPs. Por lo anterior, es de suma importancia revisar los resultados con los que se cuenta hasta el momento, ya que uno de los temas científicos de gran relevancia es la creación de biomateriales odontológicos basados en NPs.

La evidencia con la que se cuenta hasta el momento sugiere que las $\mathrm{NPs}_{\text {de }} \mathrm{TiO}_{2}$ en cultivos de células de la cavidad oral no producen un efecto citotóxico y al parecer estimulan el crecimiento celular a bajas concentraciones (hormesis). Este comportamiento puede ser atribuido a la aǵlomeración de las NPs al entrar en contacto con el cultivo celular. De manera general, en los resultados de los estudios en células in vitro se observa cierto grado de biocompatibilidad, siendo un paso importante en el campo científico encaminado al uso de las $\mathrm{NPs}$ de $\mathrm{TiO}_{2}$ en odontología que debe se ser extrapolado a un sistema in vivo tomado un cuenta todas las condiciones inherentes para realizar estos estudios.

Por otro lado, la investigación clínica sugiere que un efecto pro-inflamatorio en fibroblastos gingivales humanos es ocasionado por el uso de NPs de $\mathrm{TiO}_{2}$ en ausencia de interleucina-1 $\beta$; $y$ podría agravarse en pacientes con enfermedades bucales inflamatorias crónicas.

\section{AGRADECIMIENTOS}

Proyecto UNAM-DGAPA, PAPIME: PE201617 y PAPIIT: IA204516. Red Farmoquímicos, CONACyT.

\section{REFERENCIAS}

1. Uskokovic V. Entering the era of nanoscience: time to be so small. J Biomed Nanotechnol 2013; 9: 1441-1470.

2. Olenin AY, Lisichkin G V. Metal nanoparticles in condensed media: preparation and the bulk and surface structural dynamics. Russ Chem Rev 2011; 80: 605-630.

3. Sayes CM, Wahi R, Kurian PA, Liu Y, West JL, Ausman KD, Warheit DB, Colvin VL. Correlating nanoscale titania structure with toxicity: a cytotoxicity and inflammatory response study with human dermal fibroblasts and human lung epithelial cells. Toxicol Sci 2006; 92: 174-185.

4. Xue C, Wu J, Lan F, Liu W, Yang X, Zeng F, Xu H. Nano titanium dioxide induces the generation of ROS and potential damage in HaCaT cells under UVA irradiation. J Nanosci Nanotechnol 2010; 10: 8500-8507.

5. Weir A, Westerhoff P, Fabricius L, Hristovski K, Von Goetz N. Titanium dioxide nanoparticles in food and personal care products. Environ Sei Technol 2012; 46: 2242-2250.

6. Yang $\mathbf{P}$, Gai S, Lin J. Functionalized mesoporous silica materials for controlled drug delivery. Chem Soc Rev 2012; 41: 3679-3698.

7. Rosenholm JM, Meinander A, Peuhu E, Niemi R, Eriksson JE, Sahlg̀ren C, Lindén M. Targeting of porous hybrid silica nanoparticles to cancer cells. ACS Nano 2008; 3: 197-206.

8. Choi Y-E, Kwak J-W, Park JW. Nanotechnology for early eancer detection. Sensors 2010; 10: 428-455.

9. Youns M, D Hoheisel J, Efferth T. Therapeutic and diagnostic applications of nanoparticles. Curr Drug Targets 2011; 12: 357-365.

10. Zhang L, Gu FX, Chan JM, Wang AZ, Langer RS, Farokhzad OC. Nanoparticles in medicine: therapeutic applications and developments. Clin Pharmacol Ther 2008; 83: 761-769. 
11. Szaciłowski K, Macyk W, Drzewiecka-Matuszek A, Brindell M, Stochel G. Bioinorganic photochemistry: frontiers and mechanisms. Chem Rev 2005; 105: 2647-2694.

12. Sul Y-T. Electrochemical growth behavior, surface properties, and enhanced in vivo bone response of $\mathrm{TiO}_{2}$ nanotubes on microstructured surfaces of blasted, screwshaped titanium implants. Int J Nanomedicine 2010; $5: 87$.

13. Patri A, Umbreit T, Zheng J, Nagashima K, Goering P, Francke-Carroll S, Gordon E, Weaver J, Miller T, Sadrieh N, McNeil S, Stratmeyer M. Enerǵy dispersive X-ray analysis of titanium dioxide nanoparticle distribution after intravenous and subcutaneous injection in mice. J Appl Toxicol 2009; 29: 662-672.

14. Wiesenthal A, Hunter L, Wang S, Wickliffe J, Wilkerson M. Nanoparticles: small and mighty. Int J Dermatol 2011; 50: 247-254.

15. Montazer M, Behzadnia A, Pakdel E, Rahimi MK, Moghadam MB. Photo induced silver on nano titanium dioxide as an enhanced antimicrobial aǵent for wool. J Photochem Photobiol B Biol 2011; 103: 207-214.

16. Lee KP, Trochimowicz HJ, Reinhardt CF. Pulmonary response of rats exposed to titanium dioxide $\left(\mathrm{TiO}_{2}\right)$ by inhalation for two years. Toxicol Appl Pharmacol 1985; 79: 179-192.

17. IARC Working Group on the Evaluation of Carcinogenic Risks to Humans. Cobalt in hard metals and cobalt sulfate, gallium arsenide, indium phosphide and vanadium pentoxide. IARC Monogr Eval Carcinog risks to humans 2006 ; 86: 1-9.

18. Thurn KT, Arora H, Paunesku T, Wu A, Brown EMB, Doty C, Kremer J, Woloschak G. Endocytosis of titanium dioxide nanoparticles in prostate cancer PC-3M cells. Nanomedicine 2011; 7 : 123-130.

19. Oberdorster G. Significance of particle parameters in the evaluation of exposuredose-response relationships of inhaled particles. Inhal Toxicol 1996; 8: 73-89.

20. Filipe P, Silva JN, Silva R, De Castro JLC, Gomes MM, Alves LC, Santus R, Pinheiro T. Stratum corneum is an effective barrier to $\mathrm{TiO}_{2}$ and $\mathrm{ZnO}$ nanoparticle percutaneous absorption. Skin Pharmacol Physiol 2009; 22: 266-275.
21. Jawad H, Boccaccini AR, Ali NN, Harding SE. Assessment of cellular toxicity of $\mathrm{TiO}_{2}$ nanoparticles for cardiac tissue engineering applications. Nanotoxicology 2011; 5: 372-380.

22. Li S-Q, Zhu R-R, Zhu H, Xue M, Sun X-Y, Yao S-D, Wang SL. Nanotoxicity of TiO (2) nanoparticles to erythrocyte in vitro. Food Chem Toxicol 2008; 46: 3626-3631.

23. Cho W-S, Duffin R, Bradley M, Megson IL, MacNee W, Lee JK, Jeong J, Donaldson K. Predictive value of in vitro assays depends on the mechanism of toxicity of metal oxide nanoparticles. Part Fibre Toxicol 2013; 10: 55.

24. Jin C-Y, Zhu B-S, Wang X-F, Lu Q-H. Cytotoxicity of titanium dioxide nanoparticles in mouse fibroblast cells. Chem Res Toxicol 2008; 21: 1871-1877.

25. García-Contreras R, Arǵueta-Figueroa L, Mejía-Rubalcava C, Jiménez-Martínez R, Cuevas-Guajardo S, Sánchez-Reyna PA, Mendieta-Zeron H. Perspectives for the use of silver nanoparticles in dental practice. Int Dent J 2011; 61: 297-301.

26. Elsaka SE, Hamouda IM, Swain M V. Titanium dioxide nanoparticles addition to a conventional ǵlass-ionomer restorative: influence on physical and antibacterial properties. J Dent 2011; 39: 589-598.

27. Moher D, Liberati A, Tetzlaff J, Altman DG, PRISMA Group. Preferred reporting items for systematic reviews and metaanalyses: the PRISMA statement. PLoS Med 2009; 6: e1000097.

28. Chen X, Mao SS. Synthesis of titanium dioxide (TiO2) nanomaterials. J Nanosci Nanotechnol 2006; 6: 906-925.

29. Sugimoto T, Zhou X, Muramatsu A. Synthesis of uniform anatase $\mathrm{TiO} 2$ nanoparticles by gel-sol method: 3. Formation process and size control. J Colloid Interface Sci 2003; 259: 43-52.

30. Sugimoto T, Zhou X. Synthesis of uniform anatase TiO2 nanoparticles by the Gel-Sol method: 2. adsorption of $\mathrm{OH}-$ ions to $\mathrm{Ti}$ $(\mathrm{OH}) 4$ gel and $\mathrm{TiO} 2$ particles. J Colloid Interface Sci 2002; 252: 347-353.

31. Sugimoto T, Okada K, Itoh H. Synthetic of uniform spindle-type titania particles by the gel-sol method. J Colloid Interface Sci 1997; 193: 140-143. 
32. Sugimoto $T$, Zhou $\mathrm{X}$, Muramatsu A. Synthesis of uniform anatase $\mathrm{TiO}_{2}$ nanoparticles by gel-sol method: 4. Shape control. J Colloid Interface Sci 2003; 259: 53-61.

33. Sugimoto $T$, Zhou $X$, Muramatsu $A$. Synthesis of uniform anatase $\mathrm{TiO} 2$ nanoparticles by gel-sol method: 1. Solution chemistry of $\mathrm{Ti}(\mathrm{OH}) \mathrm{n}(4-\mathrm{n})+$ complexes. J Colloid Interface Sci 2002; 252: 339-346.

34. Su C, Hong B-Y, Tseng C-M. Sol-gel preparation and photocatalysis of titanium dioxide. Catal Today 2004; 96: 119-126.

35. Wetchakun N, Phanichphant S. Effect of temperature on the degree of anatase-rutile transformation in titanium dioxide nanoparticles synthesized by the modified sol-gel method. Curr Appl Phys 2008; 8: 343-346.

36. Do Kim K, Kim SH, Kim HT. Applying the Taguchi method to the optimization for the synthesis of $\mathrm{TiO}_{2}$ nanoparticles by hydrolysis of TEOT in micelles. Colloids Surfaces A Physicochem Enǵ Asp 2005; 254: 99-105.

37. Lin J, Lin Y, Liu P, Meziani MJ, Allard LF, Sun Y-P. Hot-fluid annealing for crystalline titanium dioxide nanoparticles in stable suspension. J Am Chem Soc 2002; 124: 11514-11518.

38. Zhang D, Qi L, Ma J, Cheng H. Formation of crystalline nanosized titania in reverse micelles at room temperature. J Mater Chem. 2002; 12: 3677-3680.

39. Lim KT, Hwang HS, Ryoo W, Johnston KP. Synthesis of $\mathrm{TiO} 2$ nanoparticles utilizing hydrated reverse micelles in $\mathrm{CO}_{2}$. Langmuir 2004; 20: 2466-2471.

40. Li Y, Lee N-H, Hwang D-S, Song JS, Lee EG, Kim S-J. Synthesis and characterization of nano titania powder with high photoactivity for gas-phase photo-oxidation of benzene from $\mathrm{TiOCl}_{2}$ aqueous solution at low temperatures. Lanǵmuir 2004; 20: 10838-10844.

41. Zane A, Zuo R, Villamena FA, Rockenbauer A, Foushee AMD, Flores K, Dutta PK, Naǵy A. Biocompatibility and antibacterial activity of nitrogen-doped titanium dioxide nanoparticles for use in dental resin formulations. Int J Nanomedicine 2016; 11: 6459.

42. Rossi S, Tirri T, Paldan H, Kuntsi-Vaattovaara H, Tulamo R, Närhi T. Peri-implant tissue response to $\mathrm{TiO} 2$ surface modified implants. Clin Oral Implants Res 2008; 19: 348-355.

43. Teubl BJ, Schimpel C, Leitinger G, Bauer B, Fröhlich E, Zimmer A, Robleǵg E. Interactions between nano- $\mathrm{TiO}_{2}$ and the oral cavity: Impact of nanomaterial surface hydrophilicity/hydrophobicity. J Hazard Mater 2015; 286: 298-305.

44. Garcia-Contreras R, Scougall-Vilchis RJ, Contreras-Bulnes R, Kanda Y, Nakajima H, Sakagami H. Induction of prostaglandin E2 production by $\mathrm{TiO}_{2}$ nanoparticles in human gingival fibroblast. In Vivo 2014; 222 : 217-222.

45. Best M, Phillips G, Fowler C, Rowland J, Elsom J. Characterisation and cytotoxic screening of metal oxide nanoparticles putative of interest to oral healtheare formulations in non-keratinised human oral mucosa cells in vitro. Toxicol in Vitro 2015; 30 : 402-411.

46. Demetrescu I, Pirvu C, Mitran V. Effect of nano-topographical features of $\mathrm{Ti} / \mathrm{TiO} 2$ electrode surface on cell response and electrochemical stability in artificial saliva. Bioelectrochemistry 2010; 79: 122-129.

47. Ma Q, Wang W, Chu P. K, Mei S, Ji K, Jin, L, Zhang Y. Concentration-and time-dependent response of human gingival fibroblasts to fibroblast growth factor 2 immobilized on titanium dental implants. Int J Nanomedicine 2012; 7: 1965-1975.

48. Giovanni M, Tay CY, Setyawati MI, Xie J, Ong CN, Fan R, Yue J, Zhang L, Leong DT. Toxicity profiling of water contextual zinc oxide, silver, and titanium dioxide nanoparticles in human oral and gastrointestinal cell systems. Environ Toxicol 2015; 30: 1459-1469.

49. Garcia-Contreras R, Scougall-Vilchis RJ, Contreras-Bulnes R, Ando Y, Kanda Y, Hibino Y, Nakajima H, Sakagami H. Effects of $\mathrm{TiO}_{2}$ nanoparticles on eytotoxic action of chemotherapeutic druǵs aǵainst a human oral squamous cell carcinoma cell line. In Vivo 2014; 28: 209-215.

50. Garcia-Contreras R, Scougall-Vilchis RJ, Contreras-Bulnes R, Kanda Y, Nakajima H, Sakagami H. Effects of $\mathrm{TiO}_{2}$ nano glass ionomer cements against normal and cancer oral cells. In Vivo 2014; 28; 895-907. 
51. Samiei M, Ghasemi N, Aghazadeh M, Divband B, Akbarzadeh F. Biocompatibility of mineral trioxide aggregate with $\mathrm{TiO}_{2}$ nanoparticles on human gingival fibroblasts. J Clin Exp Dent 2017; 9: 182-185.

52. Kroll A, Pillukat MH, Hahn D, Schnekenburger $\mathbf{J}$. Interference of engineered nanoparticles with in vitro toxicity assays. Arch Toxicol 2012; 86: 1123-1136.

53. Garcia-Contreras R, Sugimoto M, Umemura N, Kaneko M, Hatakeyama Y, Soga T, Tomita M, Scougall-Vilchis RJ, Contreras-Bulnes R, Nakajima H. Alteration of metabolomic profiles by titanium dioxide nanoparticles in human gingivitis model. Biomaterials 2015; 57: 33-40.
54. Teubl B, Leitinger G, Schneider M, Lehr CM, Fröhlich E, Zimmer A, Roblegg E E. The buccal mucosa as a route for $\mathrm{TiO}_{2}$ nanoparticle uptake. Nanotoxicol 2015; 9: 253-261.

55. Mano SS, Kanehira K, Taniguchi A. Comparison of cellular uptake and inflammatory response via toll-like receptor 4 to lipopolysaccharide and titanium dioxide nanoparticles. Int J Mol Sei 2013; 14: 13154-13170.

56. Beckonert O, Keun HC, Ebbels TMD, Bundy J, Holmes E, Lindon JC, Nicholson JK. Metabolic profiling, metabolomic and metabonomic procedures for NMR spectroseopy of urine, plasma, serum and tissue extracts. Nat Protoc 2007; 2: 2692. 